\title{
Cardiovascular nanomedicine: the route ahead
}

\author{
Michele lafisco ${ }^{1}$, Alessio Alogna², Michele Miragoli ${ }^{3,4,5}$ \& Daniele Catalucci*,4,5 (D) \\ ${ }^{1}$ National Research Council, Institute of Science \& Technology for Ceramics, Faenza, RA 48018, Italy \\ ${ }^{2}$ Medizinische Klinik m. S. Kardiologie Charité, Berlin 13353, Germany \\ ${ }^{3}$ Department of Medicine \& Surgery, University of Parma, Parma 43126, Italy \\ ${ }^{4}$ Humanitas Clinical \& Research Center, IRCCS, Rozzano, MI 20090, Italy \\ ${ }^{5}$ National Research Council, Institute of Genetic \& Biomedical Research - UOS Milan, Milan 20139, Italy \\ *Author for correspondence: Tel.: +39 0282245210; daniele.catalucci@cnr.it
}

\begin{abstract}
"Nanomedicine is well suited to address the main issues of several diseases, providing the unique possibility to design precise nanoparticles (NPs) as carriers for controlled and targeted delivery of therapeutic compounds to the site of interest"
\end{abstract}

First draft submitted: 4 June 2019; Accepted for publication: 4 July 2019; Published online: 28 August 2019

Keywords: calcium phosphate nanoparticle $\bullet$ cardiovascular disease $\bullet$ drug delivery $\bullet$ heart $\bullet$ heart failure $\bullet$ inhalation • therapeutic approach

Heart failure, coronary cardiac disease, myocardial infarction and inflammatory heart disease, along with other cardiovascular diseases (CVDs), account for the widest health problems the world over. In Europe, $45 \%$ of all deaths are due to CVDs, accounting for 3.9 million deaths per year [1]. Financially, the direct healthcare related to CVD treatment is estimated to cost the EU economy $€ 111$ billion a year, with a $€ 210$ billion yearly rate for the overall management of CVDs. Unfortunately, these figures are expected to grow over the next 10 years due to an increase in CVD risk factors, such as obesity and diabetes, as well as an expansion of the geriatric population. Thus, despite promising benefits from the recent increase in therapeutic options and pharmacological advancements (e.g., sacubitril/valsartan), the identification of new and more efficient treatment modalities is still critically required. Nanomedicine, which is among the fastest emerging research areas, is expected to fill this gap by revolutionizing the CVD care system.

\section{State-of-the-art nanomedicine for CVD treatment}

Nanomedicine is well suited to address the main issues of several diseases, providing the unique possibility to design precise nanoparticles (NPs) as carriers for controlled and targeted delivery of therapeutic compounds to the site of interest. Oncology nanomedicine was first to set the stage for this innovative approach $(\sim 7000$ hits for 'oncology' and 'nanomedicine' on PubMed) [2] and several nanomedical products (e.g., Doxil ${ }^{\circledR}$, Abraxane $^{\circledR}$ and AmBisome ${ }^{\circledR}$ ) have been approved by the US FDA and the EMA for their clinical use in humans [3-5]. The field of cardiovascular nanomedicine $(\mathrm{CVN})$ started later than that of oncology, but is now supported by a significant increase in promising lab-scale results ( $\sim 1200$ hits for 'cardiovascular' and 'nanomedicine' on PubMed) and focused toward the establishment of innovative solutions for the challenge of current CVD treatments. The majority of the first nanosystems in CVN were designed for improving the bioavailability, stability and safety of already existing drugs. For example, lipid-based NPs, which show low side effects and greater ability to passively accumulate at tissues with higher vascular permeability (enhanced permeation rate), have been largely used since the earlier times of CVN. Lipid-based nanocarriers solved the issue with the drug Wortmannin in clinical development, which was due to unmet solubility and chemical requirements [6]. A similar approach was adopted for improving the bioavailability of the clinically available carvedilol, a $B$-adrenergic receptor blocker with poor solubility [7], and Liprostin (Phase III clinical trial) [8], used for the treatment of peripheral artery disease. Improved drug solubility has also been achieved with dendrimeric formulations in complex with candesartan, a clinically approved angiotensin receptor blocker [9]. Interesting results have also been obtained with nanomedicine-based formulations loaded with omega-3 
polyunsaturated fatty acids ( $\omega-3$ PUFAs), well-known dietary factors with beneficial properties for the prevention of CVDs [10]. Polymeric NPs, silica NPs, carbon nanotubes, polymeric micelles, quantum dots, nanofibers and nanocrystals represent other examples of nano-formulations for controlled drug delivery (refer to [11,12] for more in-depth details).

In addition to gained solubility, NP-loaded drugs are expected to be protected from systemic degradation, show reduced toxicity and immunogenicity, possess ameliorated pharmacokinetics and increased half-life and exhibit increased bioavailability and precise biodistribution (refer to [13] for more in-depth details). With the possibility to be functionalized with different classes of targeting moieties, nanodrug formulations are expected to enhance selective delivery to the site of interest and benefit from a lower clearance from the body [14]. Nevertheless, the persistence of multiple technical, ethical and regulatory bottlenecks, partly due to the increasing complexity of recent nanomedical products, has hampered the effective advancement toward translational CVN. In fact, less than 20 clinical trials are currently registered at ClinicalTrials.gov (searched terms 'nanoparticle' and 'cardiovascular') and as of yet no CVN products have been approved by the FDA and EMA. In addition, despite great interest in the direct treatment of the diseased heart, the current CVN trials remain limited toward the monitoring, detection and treatment of atherosclerosis (e.g., BLAST, NANOM, Nano-Athero), occurring in the vasculature.

\section{New horizon for cardiac nanomedicine}

A disease-driven rather than a formulation-driven approach might be the choice for the appropriate nanomaterial in CVN. In addition, the selection of a proper route of drug administration (now mainly at oral and intravenous levels) might provide the CVN nano-based drug system with enhanced support for a higher benefit-to-risk ratio. Within the European Commission-funded CUPIDO project (www.cupidoproject.eu), the therapeutic targeting of the heart is tackled via small, biocompatible and inhalable NPs. The first hint for the lung-to-heart phenomenon derives from studies on combustion-derived ultrafine $(<0.1 \mu \mathrm{m}) \mathrm{NPs}$, linking air pollution to an increasing number of CVD deaths [15]. Based on the same 'toxic-driven' mechanism, the CUPIDO network exploited the possibility to 'reinvent' the NP players and generated bioinspired and self-assembling calcium phosphate nanoparticles (CaPs) [16], featuring a high degree of biocompatibility and biodegradability, and designed for crossing biological barriers, such as the one at the pulmonary level [17]. Although inhalation of nano-formulations for the treatment of pulmonary diseases has been successfully reported (e.g., ARIKACE, Pulmaquin) [18], no evidence has been provided for its novel application as a noninvasive and user-friendly approach for the direct targeting and therapeutic management of the diseased heart. The first proof-of-concept supporting this unconventional approach was recently published by the CUPIDO network, highlighting the potential for a faster, more efficient and heart-specific nanodrug administration approach compared with the traditional intravenous and oral routes. In a mouse model of diabetic cardiomyopathy, inhalation of CaPs loaded with a therapeutic mimetic peptide [19] resulted in restoration of cardiac function [17]. In addition, preliminary evidence for efficient lung-to-heart $\mathrm{CaP}$ delivery in a porcine large animal model has opened up opportunities for further translation toward clinical CVN [17].

Nanodrugs are intended to prevent adverse side effects and ligand-targeting or receptor-mediated targeting approaches refer to an additional level of complexity that might be applied to the NP product in CVN [20]. In fact, with the aim to reduce off-target toxicity issues and further implement the therapeutic selectivity to the heart, surface functionalization with cell-specific targeting groups (e.g. peptide, aptamers) may allow for a more direct and selective tuning of NP biodistribution toward the diseased myocardium. With the establishment of a further point-of-interaction with the targeted cells, more efficient NP internalization to the cell and thus enhanced release of the payload to cardiomyocytes may be envisaged. However, despite attempts by many research groups and pharmaceutical companies, no clinically approved drugs have so far been obtained for this purpose. A likely pitfall is undesirable in vivo physicochemical changes that NP may be subjected to following functionalization with the targeting ligand.

Finally, stimuli-responsive NPs (e.g., magnetic ones), might provide an additional strategy for enhanced and controlled guidance of NPs to the site of interest [21] as well as for further implementation of localized release of the NP-loaded drug [22]. In line with this, despite no clinical trial having yet passed for CVN, the NanoTherm therapy (MagForce AG) in oncology medicine represents the first magnetic NPs approved for local hyperthermia treatment of solid tumors. 


\section{From bench to clinic: a journey of translational research}

Many nanoformulations for CVN have been generated and tested in vitro and in vivo for their beneficial effects in several preclinical trials. Nevertheless, their effective translational use toward clinical development is still on the way, facing multiple challenges related to NP drug loading and release efficiency, biocompatibility and stability in biological environments as well as feasibility of pharmaceutical scale-up. As such, deepening the knowledge and maturation of the product is still required for a successful compliance with strong regulatory requirements concerning safety, stability, efficacy and Good Manufacturing Practice-compliant production (refer to [23] for full detailed description). In addition, due to the rapid development of many NPs potentially translatable into viable clinical products, continuous communication and collaboration between experts involved in all stages from academia to industry to regulatory bodies is critically required.

In conclusion, the application of CVN in patients is still in its infancy and a great effort will likely be needed for a clinical breakthrough. Biocompatible and biodegradable NPs with the synergistic combination of more efficient administration strategies, in other words, inhalation, might represent a captivating approach for the pharmacological treatment of heart failure.

Financial \& competing interests disclosure

The authors acknowledge H2020-NMBP-2016 720834 CUPIDO (www.cupidoproject.eu). The authors have no other relevant affiliations or financial involvement with any organization or entity with a financial interest in or financial conflict with the subject matter or materials discussed in the manuscript apart from those disclosed.

No writing assistance was utilized in the production of this manuscript.

\section{References}

Papers of special note have been highlighted as: $\bullet$ of interest; $\bullet \bullet$ of considerable interest

1. Wilkins EWL, Wickramasinghe K, Bhatnagar P et al. European Cardiovascular Disease Statistics 2017. European Heart Network, Brussels (2017).

2. Wolfram J, Ferrari M. Clinical cancer nanomedicine. Nano Today 25, 85-98 (2019).

-. Benefit, critical obstacles and strategies in nanomedicine reviewed in this paper.

3. Wolfram J, Zhu M, Yang Y et al. Safety of nanoparticles in medicine. Curr. Drug Targets 16(14), 1671-1681 (2015).

4. Etheridge ML, Campbell SA, Erdman AG, Haynes CL, Wolf SM, McCullough J. The big picture on nanomedicine: the state of investigational and approved nanomedicine products. Nanomedicine 9(1), 1-14 (2013).

5. Bobo D, Robinson KJ, Islam J, Thurecht KJ, Corrie SR. Nanoparticle-based medicines: a review of FDA-approved materials and clinical trials to date. Pharm. Res. 33(10), 2373-2387 (2016).

- Approved nanomaterial in the market.

6. Karve S, Werner ME, Sukumar R et al. Revival of the abandoned therapeutic wortmannin by nanoparticle drug delivery. Proc. Natl Acad. Sci. USA 109(21), 8230-8235 (2012).

7. Venishetty VK, Chede R, Komuravelli R, Adepu L, Sistla R, Diwan PV. Design and evaluation of polymer coated carvedilol loaded solid lipid nanoparticles to improve the oral bioavailability: a novel strategy to avoid intraduodenal administration. Coll. Surf. B. Biointerf. 95 , 1-9 (2012).

8. Bulbake U, Doppalapudi S, Kommineni N, Khan W. Liposomal formulations in clinical use: an updated review. Pharmaceutics 9(2), 12-45 (2017).

9. Gupta U, Agashe HB, Asthana A, Jain NK. Dendrimers: novel polymeric nanoarchitectures for solubility enhancement. Biomacromolecules 7(3), 649-658 (2006).

10. Serini S, Cassano R, Trombino S, Calviello G. Nanomedicine-based formulations containing omega-3 polyunsaturated fatty acids: potential application in cardiovascular and neoplastic diseases. Int. J. Nanomed. 14, 2809-2828 (2019).

11. Chandarana M, Curtis A, Hoskins C. The use of nanotechnology in cardiovascular disease. Appl. Nanosci. 8(7), 1607-1619 (2018).

12. Nadimi AE, Ebrahimipour SY, Afshar EG et al. Nano-scale drug delivery systems for antiarrhythmic agents. Eur. J. Med. Chem. 157 , 1153-1163 (2018).

13. Martin Gimenez VM, Kassuha DE, Manucha W. Nanomedicine applied to cardiovascular diseases: latest developments. Ther. Adv. Cardiovasc. Dis. 11(4), 133-142 (2017).

14. Dormont F, Varna M, Couvreur P. Nanoplumbers: biomaterials to fight cardiovascular diseases. Materials Today 21(2), 122-143 (2018).

- Nanomedicine in atherosclerosis reviewed in this paper.

15. Rajagopalan S, Al-Kindi SG, Brook RD. Air pollution and cardiovascular disease: JACC state-of-the-art review. J. Am. Coll. Cardiol.72(17), 2054-2070 (2018). 
16. Di Mauro V, Iafisco M, Salvarani $\mathrm{N}$ et al. Bioinspired negatively charged calcium phosphate nanocarriers for cardiac delivery of microRNAs. Nanomedicine 11(8), 891-906 (2016).

17. Miragoli M, Ceriotti P, Iafisco M et al. Inhalation of peptide-loaded nanoparticles improves heart failure. Sci. Translat. Med. 10(424), (2018).

-. The unconventional inhalation route for the delivery of drug-loaded nanoparticles to the myocardium is in this paper

18. Elhissi A. Liposomes for pulmonary drug delivery: the role of formulation and inhalation device design. Curr. Pharmaceut. Design 23(3), 362-372 (2017).

19. Rusconi F, Ceriotti P, Miragoli M et al. Peptidomimetic targeting of $\mathrm{Ca}_{\mathrm{v}}$ beta2 overcomes dysregulation of the L-type calcium channel density and recovers cardiac function. Circulation 134(7), 534-546 (2016).

20. Kamaly N, Xiao Z, Valencia PM, Radovic-Moreno AF, Farokhzad OC. Targeted polymeric therapeutic nanoparticles: design, development and clinical translation. Chem. Soc. Rev. 41(7), 2971-3010 (2012).

21. Zhang Y, Li W, Ou L et al. Targeted delivery of human VEGF gene via complexes of magnetic nanoparticle-adenoviral vectors enhanced cardiac regeneration. PloS ONE 7(7), e39490 (2012).

22. Marrella A, Iafisco M, Adamiano A et al. A combined low-frequency electromagnetic and fluidic stimulation for a controlled drug release from superparamagnetic calcium phosphate nanoparticles: potential application for cardiovascular diseases. J. Royal Soc. Interf./ The Royal Soc.15(144), (2018).

23. Cicha I, Chauvierre C, Texier I et al. From design to the clinic: practical guidelines for translating cardiovascular nanomedicine. Cardiovasc. Res. 114(13), 1714-1727 (2018).

-. Main translational steps and challenges that cardiovascular nanomedicine faces along the development of a product from bench to bedside. 